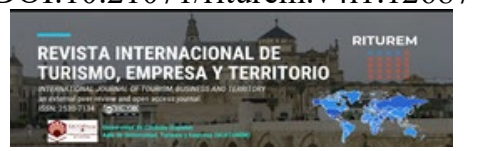

Cita bibliográfica: Pasciaroni, C., Silvina, E. y Vander, V. (2020). Innovación en hotelería: Yield Management en los hoteles de un centro urbano orientado al turismo de negocios en Argentinas. Revista Internacional de Turismo, Empresa y Territorio, 4 (1), 104-123. https://doi.org/10.21071/riturem.v4i1.12687

\title{
Innovación en hotelería: Yield Management en los hoteles de un centro urbano orientado al turismo de negocios en Argentina
}

\section{Innovation in hospitality: Yield Management in the hotels of an urban centre oriented to business tourism of Argentina}

\author{
Carolina Pasciaroni ${ }^{1^{*}}$ \\ Elías Silvina ${ }^{2}$ \\ Victoria Vander Tuin ${ }^{3}$
}

\begin{abstract}
Resumen
En la actualidad la industria hotelera se ve inmersa en un sistema de comercialización dinámico en el que las TICs (Tecnologías de la Comunicación y la Información) constituyen la base de la innovación y de las mejoras en la gestión en hotelería. En particular, el uso de sistemas automatizados para la fijación de tarifas se enmarca dentro de la implementación de una técnica específica como es el Yield Management (YM). El trabajo analiza el uso de la técnica YM en los hoteles de tres y cuatro estrellas localizados en una ciudad tamaño medio de Argentina, nodo regional de servicios, transporte y comunicación. El estudio se centra en la aplicación de la técnica YM en el marco de la política de fijación de tarifas de los hoteles. Para ello se realizan entrevistas semi-estructuradas al personal encargado de los hoteles para obtener información primaria acerca de diversos aspectos relacionados con el conocimiento e implementación efectiva de esta técnica. Factores relativos a la demanda, organizacionales y de entorno afectan la aplicación de esta técnica.
\end{abstract}

Palabras clave: gestión hotelera; innovación; fijación de tarifas; Yield Management, Argentina.

\begin{abstract}
The hotel industry is currently immersed in a dynamic marketing system in which ICTs are the basis for innovation and improvements in hotel management. The Yield Management (YM) is a technique that uses automated systems for setting prices in order to improve the hotel management processes. This paper analyses the use of the YM technique in three and four-star hotels located in a medium-sized city in Argentina, which is considered regional centre for services, communication and transport. The study focuses on the application of the YM technique in the framework of the hotel pricing-setting policy. For this purpose, semi-structured interviews are carried out to the hotel personnel in charge to obtain primary information about different features related to previous knowledge and effective implementation of this technique. Demand, organizational and environmental factors affect the adoption of this technique.
\end{abstract}

Keywords: hotel management; innovation; price setting; Yield Management, Argentina.

\footnotetext{
${ }^{1}$ Docente del Departamento de Economía, Universidad Nacional del Sur, San Andrés 800, B8002 Bahía Blanca, Buenos Aires, Argentina. carolina.pasciaroni@uns.edu.ar* Autor para la correspondencia

${ }^{2}$ Docente del Departamento de Economía, Universidad Nacional del Sur, San Andrés 800, B8002 Bahía Blanca, Buenos Aires, Argentina. selias@uns.edu.ar

${ }^{3}$ Licenciada en Turismo por el Departamento de Geografía y Turismo de la Universidad Nacional del Sur, Bahía Blanca, Buenos Aires, Argentina. vandertuin_victoria@outlook.com
} 


\section{Introducción}

En la actualidad la industria hotelera se ve inmersa en un sistema de comercialización dinámico en el que la innovación adquiere un rol destacado como fuente de competitividad y diferenciación. En esta dirección, las TICs (Tecnologías de la Comunicación y la Información) constituyen la base de la innovación y de las mejoras en la gestión en hotelería. Las TICs permiten almacenar y analizar datos, y en consecuencia, alcanzar un mejor posicionamiento en el mercado. De esta forma, y gracias a la tecnología, se puede mejorar la gestión empresarial y la toma de decisiones respecto a la fijación de tarifas. Es así como fueron apareciendo interrogantes acerca de cuánto y cuándo debían modificarse las tarifas para optimizar las ventas y mejorar la rentabilidad hotelera. Los precios cambian constantemente a tiempo real y, por este motivo, contar con sistemas computarizados cada vez más eficientes permite que las necesidades de los clientes sean atendidas con mayor precisión. De esta forma $\mathrm{y}$, contando con un buen funcionamiento de dichos sistemas, los ingresos del hotel aumentarían significativamente.

Frente a lo expuesto, el uso de sistemas automatizados para la fijación de tarifas se enmarca dentro de la implementación de una técnica específica como es el Yield Management (YM), también denominado en la literatura como Revenue Management (RM). Esta técnica consiste en la asignación a la unidad correcta de capacidad (asiento de un avión, habitación de un hotel, etc.) del precio correcto y al cliente correcto, de forma que se consiga el máximo beneficio posible (Smith, Leimkuhler y Darrow, 1992). La técnica YM es utilizada en todo el mundo por empresas de servicios con capacidad fija, tales como las empresas hoteleras, y en los últimos años su uso ha crecido gracias a los beneficios que genera al maximizar los ingresos de una empresa. Esto significa que, si quieren vender sus habitaciones a la tarifa más alta, los hoteles se encontrarán con la dificultad de que no podrán cubrir todas las plazas o, si por el contrario abaratan sus habitaciones, perderán ingresos que podrían haber obtenido de aquellos clientes dispuestos a pagar las tarifas más elevadas por la prestación de un buen servicio (Kimes, 1989). Por otra parte, la industria hotelera se enfrenta a un entorno cada vez más competitivo, motivo por el cual resulta cada vez más complejo fijar un precio según el canal, el producto, la fecha y el cliente adecuado. A modo de ejemplo, numerosas variables como el momento del año, el horario del vuelo, el día de la semana, entre otras influyen en la fijación de tarifas. Estos factores dificultan la tarea y, por ello, resulta necesario contar con un sistema de información que asigne correctamente el número de plazas a vender a la tarifa correcta. Por este motivo, hoy en día el YM cumple un rol fundamental en vistas a aumentar la rentabilidad del hotel y cuidar las preferencias de los clientes, como así también a mantenerlos leales en el tiempo.

Tomando como punto de partida la relevancia que adquieren las innovaciones basadas en el uso de las TICs en el sector hotelero, específicamente aquellas asociadas a la gestión del ingreso y la fijación de tarifas, el presente estudio tiene como objetivo analizar el uso de la técnica YM por parte de los hoteles de tres y cuatro estrellas de la ciudad de Bahía Blanca (Argentina). Si bien el estudio de la técnica YM tiene distintas dimensiones de análisis (ver Ivanov, 2014, p. 12), el trabajo propuesto se centra en el uso de la técnica YM para la fijación de tarifas, su grado de conocimiento y uso entre los hoteles, el empleo de softwares específicos, la afectación de personal idóneo, y los obstáculos/inconvenientes percibidos.

Por otra parte, ciertos aspectos asociados al área de estudio fundamentan su estudio. Por un lado, la relevancia que el sector de alojamiento tiene dentro de la industria turística nacional. Según la Encuesta de Ocupación Hotelera (EOH) de abril de 2019, se estimaron 3.5 millones de pernoctaciones en establecimientos hoteleros y para-hoteleros, lo que implica $1.6 \%$ de incremento respecto al mismo mes del año anterior (INDEC, 2019). A su vez, tal como señaló el secretario de Turismo de la Nación, Gustavo Santos en al año 2019: "el turismo sigue consolidando su aporte a la economía nacional, generando puestos de trabajo y condiciones para 
el arraigo en toda la Argentina"4. Por otro lado, Bahía Blanca es una ciudad de tamaño medio localizada en el sudoeste de la Provincia de Buenos Aires que se desempeña como un centro político-administrativo comercial, financiero y de servicios de salud y educativos para una amplia zona de influencia, a la vez que, se constituye como un nodo de comunicación y transporte y centro de escala y distribución de turistas y visitantes hacia los centros turísticos de playa y montaña cercanos. Es preciso destacar que entre la variada oferta de alojamiento de la ciudad, los hoteles locales de 3 y 4 estrellas no pertenecen a cadenas internacionales, sino que se trata de hoteles independientes y hoteles pertenecientes a cadenas de origen y alcance nacional.

Se espera que el estudio propuesto, de carácter exploratorio y descriptivo, pueda contribuir al conocimiento empírico sobre la implementación de la técnica YM en hotelería. En primer lugar, la literatura empírica sobre las restricciones e inconvenientes que afrontan los hoteles derivados de la implementación de YM son menos numerosos en comparación con otras dimensiones de la técnica (Chávez Miranda, Ruiz Jiménez y Pérez Díez de los Ríos, 2014, p. 29). En segundo lugar, los estudios sobre el grado de difusión de la técnica YM y los obstáculos asociados al uso de la misma resultan aún menos frecuentes si se adopta como caso de estudio a los hoteles que no pertenecen a grandes cadenas internacionales y que se encuentran localizados en países de menor grado de desarrollo. En este sentido, tal como se expondrá en la última sección, surgen condicionantes propios del contexto donde se desempeñan los hoteles bajo estudio, los cuales no responden a las políticas de gestión uniformes que aplican las cadenas internacionales.

A continuación se revisa la literatura referida a innovación en hotelería, específicamente, los aportes relativos a técnica YM. El artículo sigue la siguiente estructura. En la segunda sección se exponen consideraciones metodológicas, el tipo de información relevada mediante entrevistas y se describe el área de estudio y los hoteles de tres y cuatro estrellas bajo análisis. En la tercera sección, se sistematiza la información primaria relevada. En la cuarta y última sección, se ofrece una síntesis de los resultados y futuros lineamientos.

\section{Revisión de la literatura}

La innovación es fuente de competitividad, diferenciación, aumento de valor agregado $\mathrm{y}$, por lo tanto, crecimiento para las empresas hoteleras en un entorno de creciente competencia. De acuerdo al Manual de Oslo, se entiende por innovación a "la introducción de un nuevo, o significativamente mejorado, producto (bien o servicio), de un proceso, de un nuevo método de comercialización o de un nuevo método organizativo, en las prácticas internas de la empresa, la organización del lugar de trabajo o las relaciones exteriores" (OECD-EUROSTAT, 2005, p. 56). El Manual de Oslo publicado por la OCDE (Organización para la Cooperación y el Desarrollo Económicos) constituye la principal guía para la recolección e interpretación de datos sobre innovación. Inicialmente, el manual refería a la innovación en el sector industrial; a partir de la segunda edición se incorporaron aspectos específicos relativos a la innovación en el sector servicios teniendo en cuenta la importancia de este sector en las economías desarrolladas. A modo de ejemplo, las innovaciones de producto en el sector servicios pueden incluir mejoras en la prestación de los mismos traducidas en mayor eficiencia y rapidez; las innovaciones de proceso se pueden manifestar en la introducción de un nuevo sistema de reservas en una agencia de viajes (OECD-EUROSTAT, 2005, p. 59-60).

Dentro del sector servicios, la innovación en el sector hotelero se encuentra ampliamente asociada a los avances en las TICs (Benckendorff, Sheldon y Fesenmaier, 2019; Fernández, Cala y Domecq, 2011; Sigala, 2003). El uso de las TICs suponen la reducción de los costos de operación y el aumento de la eficiencia y calidad de los servicios prestados (Benckendorff et

\footnotetext{
4 Extraído del sitio: https://www.argentina.gob.ar/turismo
} 
al., 2019). La aplicación de las TICs en el sector hotelero se refiere a los siguientes campos: aplicaciones front-office (sistemas de reserva, check-in y check-out, estado y gestión de mantenimiento de las habitaciones); aplicaciones back-office (personal, módulos de compra e inventarios, módulos contables); aplicaciones de interface asociadas a la relación con el huésped (sistemas de contabilidad de llamadas, bloqueo electrónico, dispositivos operativos para huéspedes relacionados con el entretenimiento, vending); sistemas de management de restaurantes y banquetes. Sin embargo, la difusión de las TICs depende del tipo de empresa hotelera en términos de tamaño y pertenencia a una cadena (Benckendorff et al., 2019.). Si bien, inicialmente, las TICs no se difundieron rápidamente en el sector hotelero, actualmente es considerado un sector intensivo en información (Law y Jogaratnam, 2005; Benckendorff et al., 2019). Es preciso destacar que las empresas no innovan solas; la innovación es un proceso que depende de la adquisición de conocimiento e información proveniente de distintos agentes, permitiendo a la firma acceder a nuevas tecnologías y nuevos mercados (OECD-EUROSTAT, 2005: Lundvall, 2010, entre otros). Al respecto, Nieves y Segarra-Ciprés (2015) encuentran que los vínculos con agentes externos influyen de forma positiva sobre la innovación en gestión en hoteles.

Siguiendo a Fernández et al. (2011), las reservas por Internet, el correo electrónico y la conexión a Internet de banda ancha en las habitaciones de hotel, fueron implementas fácilmente y resultaron en una mejora inmediata en calidad e ingresos, además de agregar un cierto prestigio al hotel. El ritmo más lento de difusión de las TICs se evidencia a la actualidad en las aplicaciones relacionadas con la estructura productiva y de gestión del negocio hotelero tales como la técnica Yield Management (YM).

De acuerdo a Jones (2000), existen varias definiciones de $\mathrm{YM}^{5}$. Tal como se indicó en la introducción, esta técnica es definida como el proceso de asignación de una unidad de capacidad al tipo de cliente adecuado, en el momento adecuado y al precio adecuado con el objetivo de maximizar el rendimiento, traduciéndose en el caso de los hoteles, en el número de habitaciones que deben venderse a distintos niveles de tarifas (Kimes, 1989, p. 15; Smith et al., 992). Posteriormente, Kimes y Chase (1998) definen al YM como la gestión de las 4 C de un servicio perecedero de forma tal de maximizar el beneficio: calendario (calendar, con cuánta antelación se realiza la reserva.); reloj (clock, el momento del día en que se ofrece el servicio); capacidad (capacity, inventario del recurso de capacidad) y demanda del cliente (customer demand); todo ello de forma que se maximice el beneficio. Por su parte, Jones (2000, p. 88), define al YM como un sistema para maximizar la rentabilidad en hoteles, identificando la rentabilidad de los segmentos de mercado, estableciendo precios, creando reglas de descuento para su aplicación en los avanzados procesos de reserva y monitoreando la efectividad de esas reglas.

La aplicación de la técnica YM resulta apropiada en sectores donde las firmas operan con capacidad relativamente fija; cuentan con la posibilidad de segmentar el mercado; poseen inventario perecedero; posibilidad de vender el producto anticipadamente; demanda variable en función del momento en el tiempo aunque predecible; costes marginales de venta bajos y costes marginales de producción altos (Kimes 1989; Kimes and Chase, 1998). El sector hotelero responde a estos requisitos, a la vez que, presenta características diferenciales respecto a las aerolíneas a considerar al momento de aplicar la técnica YM, tales como, estancias de múltiples noches; efecto multiplicador entre los servicios de alojamiento y servicios de restauración; mayor tiempo de antelación de realización de las reservas en comparación con las aerolíneas; diferentes horarios de arribo por parte de los clientes (Jones, 2000, ver revisión Chávez Miranda, 2005).

\footnotetext{
5 Para una completa revisión de la literatura sobre YM desde los años 80s ver Anderson y Xie (2010).
} 
La técnica YM se basa en el uso de la información y por lo tanto en la aplicación de soluciones informáticas para su procesamiento. En el caso de la empresa hotelera, con el objetivo último de maximizar la rentabilidad, esta técnica utiliza información histórica pasada y niveles actuales de ocupación para pronosticar la demanda de una forma más precisa y así poder fijar y adaptar la toma de decisiones sobre tarifas y disponibilidad de habitaciones, en los diferentes canales de venta (Cheraghi, Dadashzadeh y Venkitachalam, 2010). Siguiendo a O'Connor y Murphy (2008), los sistemas computarizados permiten incorporar más factores y realizar análisis más sofisticados en base al rendimiento pasado y los patrones de reserva actuales, las tendencias del mercado. Al respecto, los hoteles empezaron a utilizar los llamados GDS (Global Distribution System), para efectuar las reservas de habitaciones y almacenar datos para poder crear un historial que pudiera predecir la demanda y analizar la competencia y, así, calcular la tarifa adecuada para cada segmento de clientes (Johnson, 2006).Sin embargo, el YM no sólo es una técnica computarizada dado que combina sistemas de información y tecnología con experiencia y conocimiento empresarial (Lieberman, 1993, p. 36 en O'Connor y Murphy, 2008).

Desde la década del 80, distintos estudios dan cuenta de la efectividad de la técnica YM en términos de mejoras en el desempeño económico en hoteles (Orkin, 1988; Lieberman, 1993, González Serrano, Talón y Figueroa, 2004; Emeksiz, Gursoy e Icoz, 2006). Para Jones (2000), implantar esta técnica en una empresa trae consigo beneficios en términos de mayor información sobre los clientes y mejoras el proceso de gestión de información manifestadas en la posibilidad de obtener estimaciones de demanda y prever los efectos de cambios en las reservas. Siguiendo a Ferguson y Smith (2014), uno de los principales beneficios secundarios derivados de adoptar prácticas de gestión de ingresos en el hotel constituye la mejora en la capacidad para pronosticar reservas futuras, sirviendo para pronosticar así el rendimiento futuro $\mathrm{y}$, por lo tanto, tomar decisiones tácticas importantes con respecto al personal, presupuestos, gastos y políticas. Por otra parte, tales prácticas, orientadas inicialmente en la gestión de la capacidad y el precio, tienen una creciente importancia en la elaboración de estrategias centradas en el cliente, tales como estrategias de ventas y marketing y gestión de los canales de distribución (Kimes, 2017; ver revisión de Erdem y Jiang, 2016).

Más allá de sus beneficios, la técnica YM no está exenta de limitantes relativos tanto a su implementación como a potenciales efectos negativos. En este último caso, un número creciente de estudios analizan la percepción de los clientes y los potenciales conflictos tras la aplicación de la técnica YM (Wirtz, Kimes, Theng y Patterson, 2003; Kimes, 2002; Choi y Mattila, 2004; Kimes y Wirtz, J., 2003; Heo y Lee, 2011). En relación a los factores que obstaculizan su implementación, en un estudio realizado a más de 160 hoteles, Okumus (2004) señala la falta de conocimiento a la escasa formación y cultura sobre la técnica, la baja adaptación de un software para su aplicación, su costo de instalación y la resistencia por parte de directivos a invertir en el proyecto de software, A estos inconvenientes se suman desafíos propios del contexto actual. Los distintos canales de distribución basados en Internet con diferentes estructuras de costos complican el proceso de decisión respecto a la fijación del precio dado que los hoteles no solo deben manipular el precio en respuesta a la oferta y la demanda, sino también debe elegir qué cartera de canales de distribución utilizar (O'Connor y Murphy, 2008; Choi y Kimes, 2002). En este contexto de creciente complejidad en la toma decisiones, se acentúa la necesidad de recursos humanos con habilidades, especialmente analíticas y comunicacionales, y puestos de trabajo en el interior de los hoteles específicamente dedicados a la gestión de ingresos (Kimes, 2017; Ferguson y Smith, 2014; Erdem y Jiang, 2016). Es debido a esto que, generalmente, los hoteles de tamaño más pequeño, debido a restricciones de costos, no pueden asignar un gerente de tiempo completo para esta función (Ferguson y Smith, 2014). 
Por último, de acuerdo a Chávez Miranda et al., 2014, los estudios de la Comisión Europea (1997) y Luciani (1999) constituyen los aportes pioneros en el análisis de los obstáculos y/o dificultades encontrados por los hoteles para la implementación del YM/RM. En su análisis referido a hoteles de 4 y 5 estrellas de Sevilla, Chávez Miranda et al. (2014) encuentran que la formación del personal y la no disposición de información interna constituyen los obstáculos iniciales. Tras la aplicación de la técnica, los obstáculos se concentran en la falta de formación oportuna por parte del personal del hotel para aplicar la técnica y la falta de un software de YM/RM adaptado totalmente al sector. El acceso a financiamiento y aspectos organizativos vinculados a la predisposición al cambio y rigideces, son factores alternativos que condicionan la decisión de innovar de las empresas hoteleras (López-Fernández, Serrano-Bedia y Gómez-López, 2009). Orfila-Sintes y Mattsson (2009) encuentran que el tamaño, la prestación de servicios adicionales, el motivo de viaje, y la capacitación de los recursos humanos tienen un efecto positivo sobre la probabilidad de obtener innovaciones back-office en los hoteles.

\section{Metodología}

El presente trabajo emplea al estudio de caso como técnica metodológica. Lo hoteles de tres y cuatro estrellas de la ciudad de Bahía Blanca (Argentina) constituye el caso de estudio seleccionado. De acuerdo a Yin (1981) el estudio de caso es una estrategia de investigación usada en las ciencias sociales para contribuir al conocimiento sobre individuos, grupos, organizaciones, fenómenos sociales, políticos. El estudio de caso es un método empírico que investiga un fenómeno contemporáneo dentro de su contexto de la vida real. Los estudios de casos no implican usar un solo tipo de evidencia, por el contrario se basan tanto en información de tipo cuantitativa como cualitativa, y su alcance puede ser exploratorio, descriptivo y explicativo (Yin, 1981). En el presente trabajo, se realizaron entrevistas semi-estructuradas presenciales en los siete hoteles que componen en su conjunto la oferta hotelera de 3 y 4 estrellas de la ciudad. Las entrevistas fueron realizadas al personal a cargo de los hoteles entre los meses de enero a marzo del $2019^{6}$.

\section{Cuadro 1. Información relevada en las entrevistas}

\begin{tabular}{|l|l|}
\hline Características hoteles & $\begin{array}{l}\text { Categoría del hotel } \\
\text { Localización en la ciudad } \\
\text { Año de Inicio de actividades } \\
\text { Número de empleados } \\
\text { Tipo de Empresa } \\
\text { Número de habitaciones y plazas }\end{array}$ \\
\hline Características de la demanda & $\begin{array}{l}\text { Motivo por el que se hospedan los clientes } \\
\text { Origen de los clientes } \\
\text { Frecuencia }\end{array}$ \\
\hline $\begin{array}{l}\text { Segmentación, overbooking y } \\
\text { sistemas de reservas }\end{array}$ & $\begin{array}{l}\text { Aplicación por parte de los hoteles de prácticas de } \\
\text { segmentación y overbooking } \\
\text { Implementación de sistema de reservas }\end{array}$ \\
\hline $\begin{array}{l}\text { Fuentes de información } \\
\text { utilizadas por el hotel para } \\
\text { actualizarse sobre fijación de } \\
\text { tarifas }\end{array}$ & $\begin{array}{l}\text { Otros hoteles (de la cadena, competencia) } \\
\text { Internet, redes sociales, revistas, ferias empresariales }\end{array}$ \\
\hline
\end{tabular}

\footnotetext{
${ }^{6}$ Para un mayor detalle de las entrevistas ver Vander Tuin (2019).
} 


\begin{tabular}{|l|l|}
\hline & $\begin{array}{l}\text { Otras instituciones/personas (Federación Empresaria } \\
\text { Hotelera Gastronómica de la Provincia de Buenos Aires } \\
\text { (FEHGPBA) y a la Federación Empresaria Hotelera } \\
\text { Gastronómica de la República Argentina (FEHGRA)) }\end{array}$ \\
\hline Conocimiento sobre YM & $\begin{array}{l}\text { Conocimiento por parte del hotel de la técnica YM más } \\
\text { allá de su aplicación efectiva }\end{array}$ \\
\hline $\begin{array}{l}\text { Aplicación efectiva de la técnica } \\
\text { YM para la fijación de tarifas }\end{array}$ & $\begin{array}{l}\text { Tiempo de implementación de la técnica } \\
\text { Razones de implementación } \\
\text { Uso de software especifico } \\
\text { Personal dedicado específicamente al uso de la técnica } \\
\text { (yield manager) } \\
\text { Obstáculos/inconvenientes percibidos por el entrevistado } \\
\text { asociados a la aplicación de la técnica YM }\end{array}$ \\
\hline $\begin{array}{l}\text { Aplicación potencial de la } \\
\text { técnica YM para la fijación de } \\
\text { tarifas }\end{array}$ & $\begin{array}{l}\text { Obstáculos/inconvenientes percibidos por el entrevistado } \\
\text { asociados a la potencial aplicación de la técnica YM }\end{array}$ \\
\hline
\end{tabular}

Elaboración propia

\subsection{Caracterización del caso de estudio}

La ciudad de Bahía Blanca se encuentra ubicada a los $38^{\circ} 44^{\prime}$ de latitud sur y $62^{\circ} 16^{\prime}$ longitud oeste, en el sudoeste de la Provincia de Buenos Aires. Según el último Censo Nacional de Población y Vivienda ${ }^{7}$, la ciudad cuenta con 301.531 habitantes, situándose en la categoría de ciudad de tamaño medio según la estructura urbana del país. Su localización geográfica, estructura productiva e infraestructura transporte y comunicaciones convierten a la ciudad en un centro de gravitación regional (Gorenstein et al., 2010; Diez, 2008). En esta dirección, la ciudad de Bahía Blanca se ha consolidado como centro comercial, financiero y de prestación de servicios de salud y educativos para una gran zona de influencia. Si tenemos en cuenta el sector industrial, se destacan un conjunto de empresas asociadas a la actividad petroquímica, agroindustrial y refinerías de petróleo. La ciudad cuenta con un puerto local, único de aguas profundas del país, que permite la salida de granos, oleaginosas hacia mercados internacionales. La ciudad se desempeña, además, como nodo de comunicaciones, conectada a las principales ciudades del país a través de un aeropuerto y una red de rutas nacionales y provinciales. A su vez, cuenta con terminal de ómnibus, terminal de trenes, transporte urbano, combis a la región y alquiler de vehículos. Por último, es sede de los consulados de Chile, España, Italia, Francia, Dinamarca y Noruega.

Desde la perspectiva del turismo, la ciudad de Bahía Blanca funciona como centro de escala más que como centro turístico de mayor permanencia. De acuerdo a la Encuesta de Ocupación Hotelera (INDEC, 2019), la estadía promedio en la ciudad es de 1,6 noches, resultando inferior a la estadía registrada por otros centros urbanos localizados en la Provincia de Buenos Aires. Siguiendo la información presentada en la página web del Municipio de Bahía Blanca, la ciudad cuenta con una amplia y variada oferta hotelera y extra-hotelera (Gráfico 1). Las plazas totales disponibles en Bahía Blanca ascienden a 1160 y están distribuidas entre hoteles, apart hoteles, hospedajes, hostels y moteles. La ciudad posee ventajas y capacidades para la organización de congresos, ferias, reuniones deportivas, comerciales y culturales y cuenta con 20 agencias de viajes y turismo habilitadas, las cuales ofrecen venta de servicios turísticos nacionales e internacionales. Asimismo en la ciudad se localiza la Asociación de

\footnotetext{
${ }^{7}$ El último Censo Nacional de Población y Vivienda corresponde al año 2010.
} 
Hoteles, Restaurantes, Bares, Confiterías y Afines de Bahía Blanca y Región Sudoeste. Si se tiene en cuenta la distancia entre los hoteles y el centro cívico de la ciudad, el $80 \%$ de los alojamientos se encuentran a menos de 10 cuadras. El $20 \%$ restante se encuentra en avenidas principales de acceso a la ciudad y en zonas más alejadas del centro (Lehr, 2018).

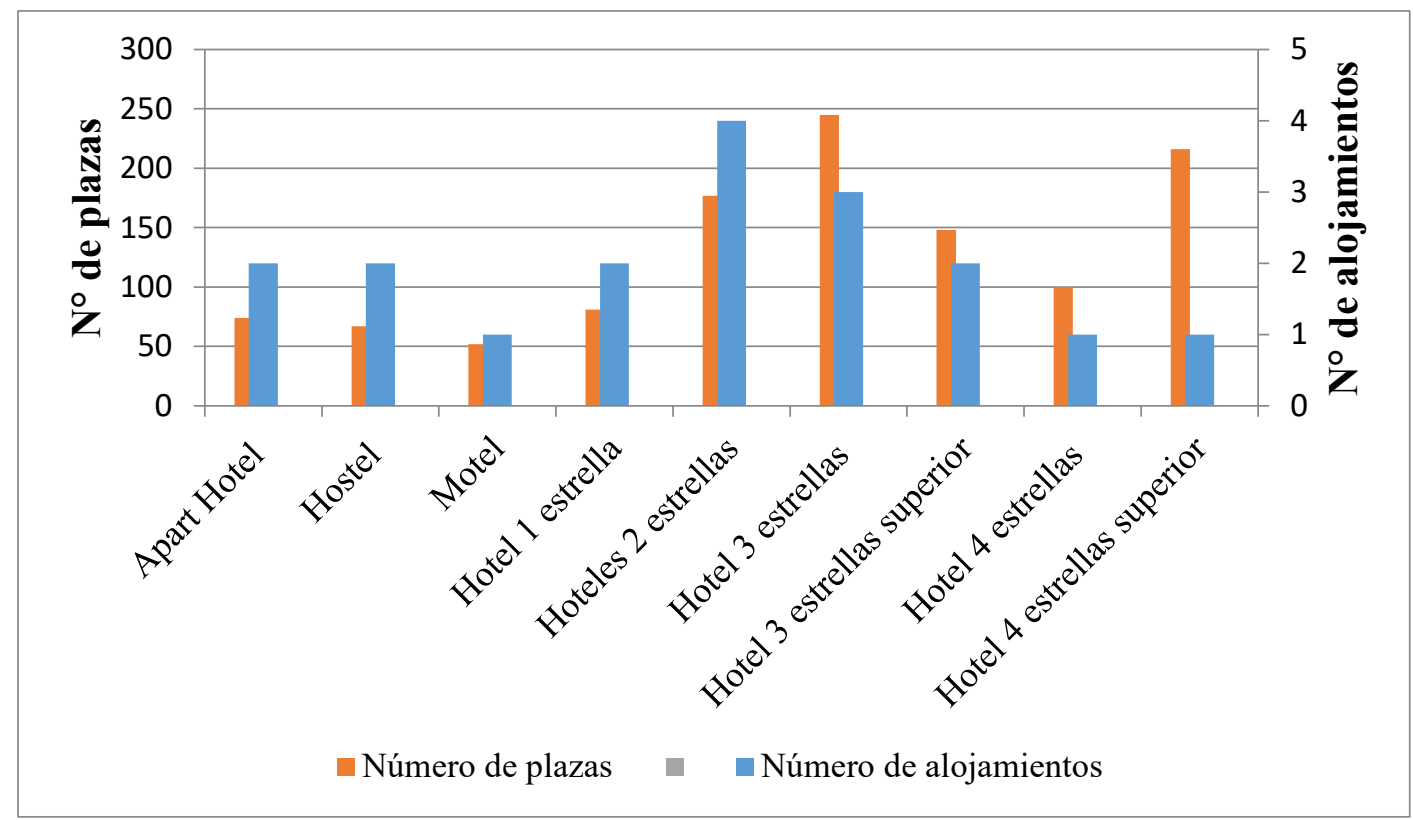

Gráfico 1. Bahía Blanca: oferta hotelera y extra-hotelera

Fuente: Elaboración propia en base a las páginas web de los alojamientos y a la página del Municipio de la ciudad de Bahía Blanca http://www.bahia.gob.ar

En relación a los hoteles bajo estudio, los siete hoteles de la ciudad de Bahía Blanca que responden a las categorías de 3 y 4 estrellas accedieron a participar de entrevistas. A los fines del análisis propuesto los hoteles fueron identificados con una única letra en orden alfabético decreciente (A - G). En conjunto, los hoteles de 3 y cuatro estrellas de la ciudad tienen las siguientes características:

- Tres de los hoteles pertenecen a la categoría de 3 estrellas, dos hoteles a la categoría tres estrellas superior, un hotel a la categoría 4 estrellas y un hotel a la categoría de 4 estrellas superior (Gráfico 2).

- Todos los hoteles, excepto dos hoteles de categoría 3 estrellas, tienen más de 40 habitaciones. El conjunto de los hoteles entrevistados alcanza como máximo las 108 habitaciones (Gráfico 2).

- La mayoría de los hoteles no forman parte de una cadena; solo tres hoteles operan bajo la modalidad de una cadena (Gráfico 2). Se trata de la cadena de Hoteles Land y la cadena Hoteles Austral, ambas de origen y alcance nacional;

- Todos los hoteles tienen amplia trayectoria, contando con más de 10 años de funcionamiento en el sector hotelero de la ciudad de Bahía Blanca. La mayoría de los hoteles responden al tipo societario S.R.L (Sociedad de Responsabilidad Limitada); solo dos hoteles responden al tipo S.A (Sociedad Anónima). 
- Los motivos por los que los clientes de los hoteles entrevistados se hospedan son principalmente por trabajo, salud, comercio y trámites. En cuanto a la procedencia de los huéspedes, todos los hoteles respondieron que, mayoritariamente, sus huéspedes son nacionales, haciendo referencia más específicamente a la Ciudad de Buenos Aires, el sur del país y la zona de influencia de Bahía Blanca como principales focos de su demanda. Por otra parte, si bien mencionaron que reciben huéspedes del exterior, la cantidad es limitada. Todos los hoteles de la ciudad coinciden en que los arribos corresponden a huéspedes frecuentes.

- Todos los hoteles de 3 y 4 estrellas de la ciudad utilizan sistemas de reservas y segmentan el mercado (Gráfico 3);

- Todos los hoteles, excepto dos, llevan a cabo la práctica de overbooking. Uno de los hoteles que no adopta esta práctica señaló que la misma atenta contra su política de fidelización de los clientes (Gráfico 3). El overbooking constituye una práctica ampliamente conocida en la industria hotelera definida como la reserva de un número de habitaciones superior a la capacidad disponible del hotel (Ivanov, 2006). También recurren a canales de distribución disponibles en Internet como Booking y Trivago.

- En cuento a los criterios para maximizar los ingresos, el análisis del nivel de ocupación es el criterio más difundido entre los hoteles bajo estudio, seguido por aumento de tarifas, atraer grupos y segmentar el mercado (Gráfico 4).

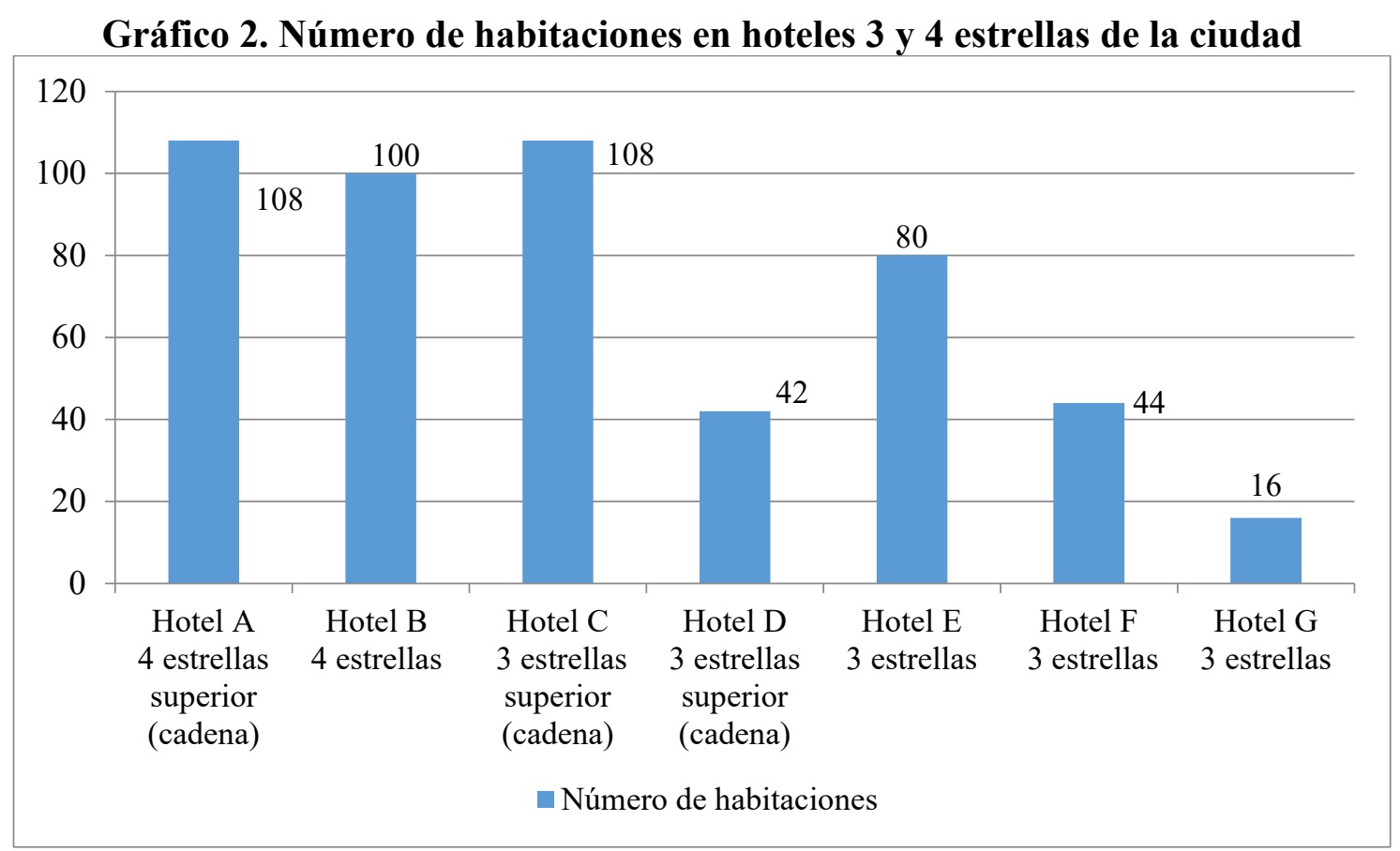

Fuente: Elaboración propia en base a datos disponibles en páginas web de los hoteles y datos suministrados en las entrevistas.

Gráfico 3. Número y porcentaje de hoteles 3 y 4 estrellas de la ciudad que segmenta el mercado, tiene sistemas de reservas y practica overbooking 


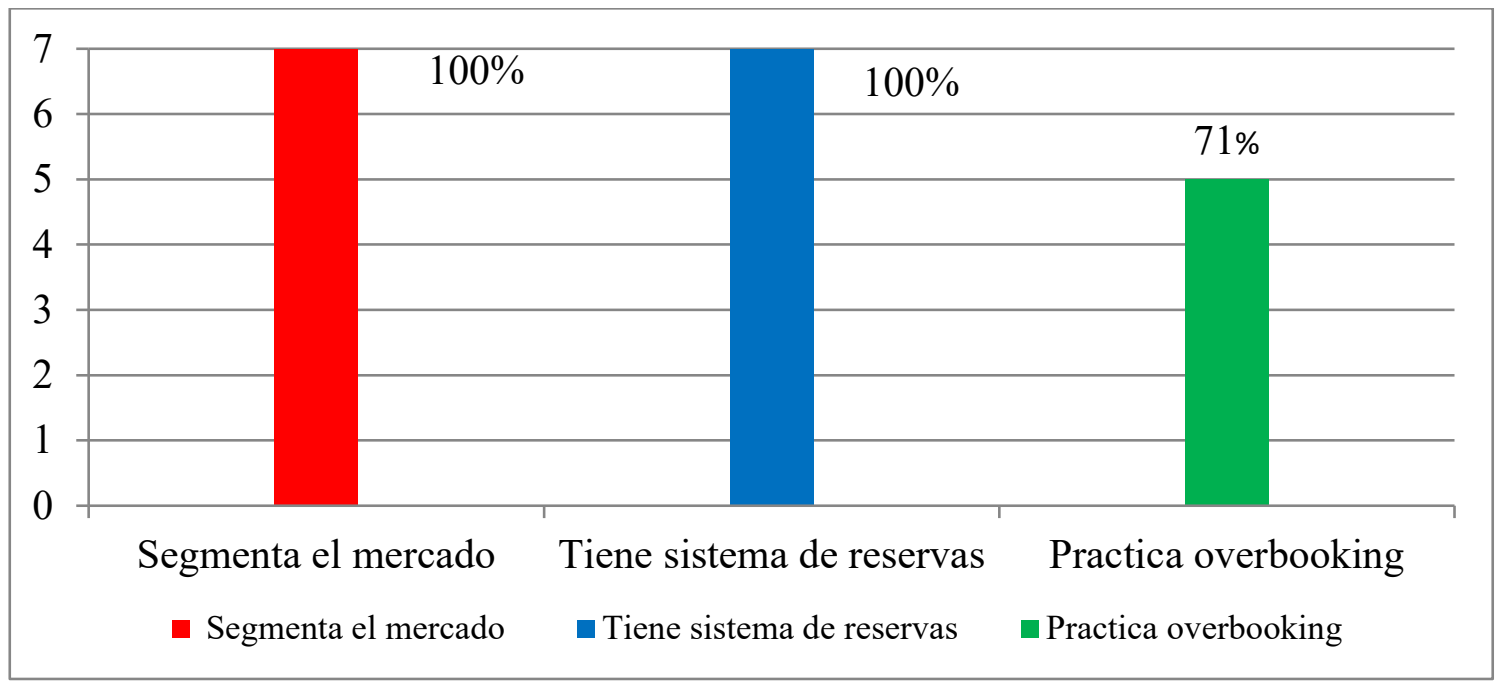

Elaboración propia

Gráfico 4. Criterios usados por los hoteles para maximizar ingresos

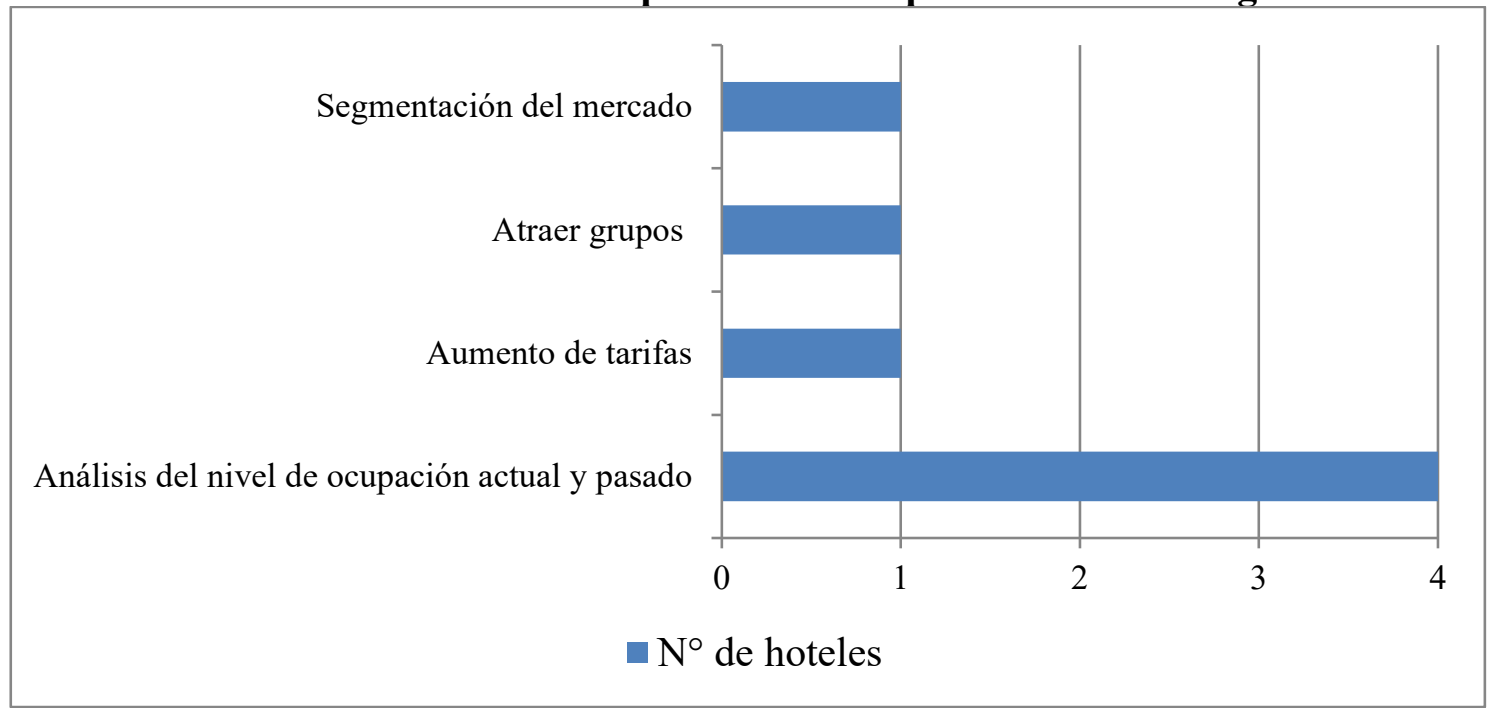

Elaboración propia

\section{Resultados: conocimiento y aplicación de la técnica Yield Management}

En esta sección se muestran los resultados relativos al 1) conocimiento del YM y las fuentes de información empleadas por los hoteles para actualizarse sobre nuevas técnicas para la fijación de tarifas y 2) la aplicación efectiva de la técnica YM. En este último punto se caracteriza a los hoteles que aplican la técnica, el tipo de software empleado, el personal afectado a la gestión del ingreso y los inconvenientes percibidos tras su implementación. El análisis se completa con la caracterización de los hoteles que no aplican la técnica YM.

En relación al conocimiento previo de los hoteles respecto a la técnica YM, de acuerdo al Gráfico 5, los hoteles de mayor categoría, a excepción del único hotel de 4 estrellas entrevistado, conocen esta técnica y recurren a distintas fuentes de información para actualizarse respecto a técnicas y procesos de fijación de tarifas. En el caso de los hoteles de 3 estrellas, sólo uno de los tres hoteles que comparten esta categoría conoce la técnica YM. De 
todos modos, los hoteles que no conocen la técnica YM manifestaron utilizar distintas fuentes de información disponible (Internet, revistas especializadas, ferias) e información proveniente de cámaras empresariales. Es preciso indicar la relevancia de estos dos últimos tipos de fuentes de información, dado que los cuatros hoteles que manifestaron conocer la técnica YM (hoteles A, C, D y G en Gráfico 5) descubrieron la misma por medio de ferias de turismo e internet. Asimismo, todos los hoteles entrevistados utilizan internet para la búsqueda de información al momento de fijar tarifas.

\section{Gráfico 5. Conocimiento sobre YM y fuentes de información sobre nuevas técnicas para la fijación de tarifas. Hoteles 3 y 4 estrellas de la ciudad.}
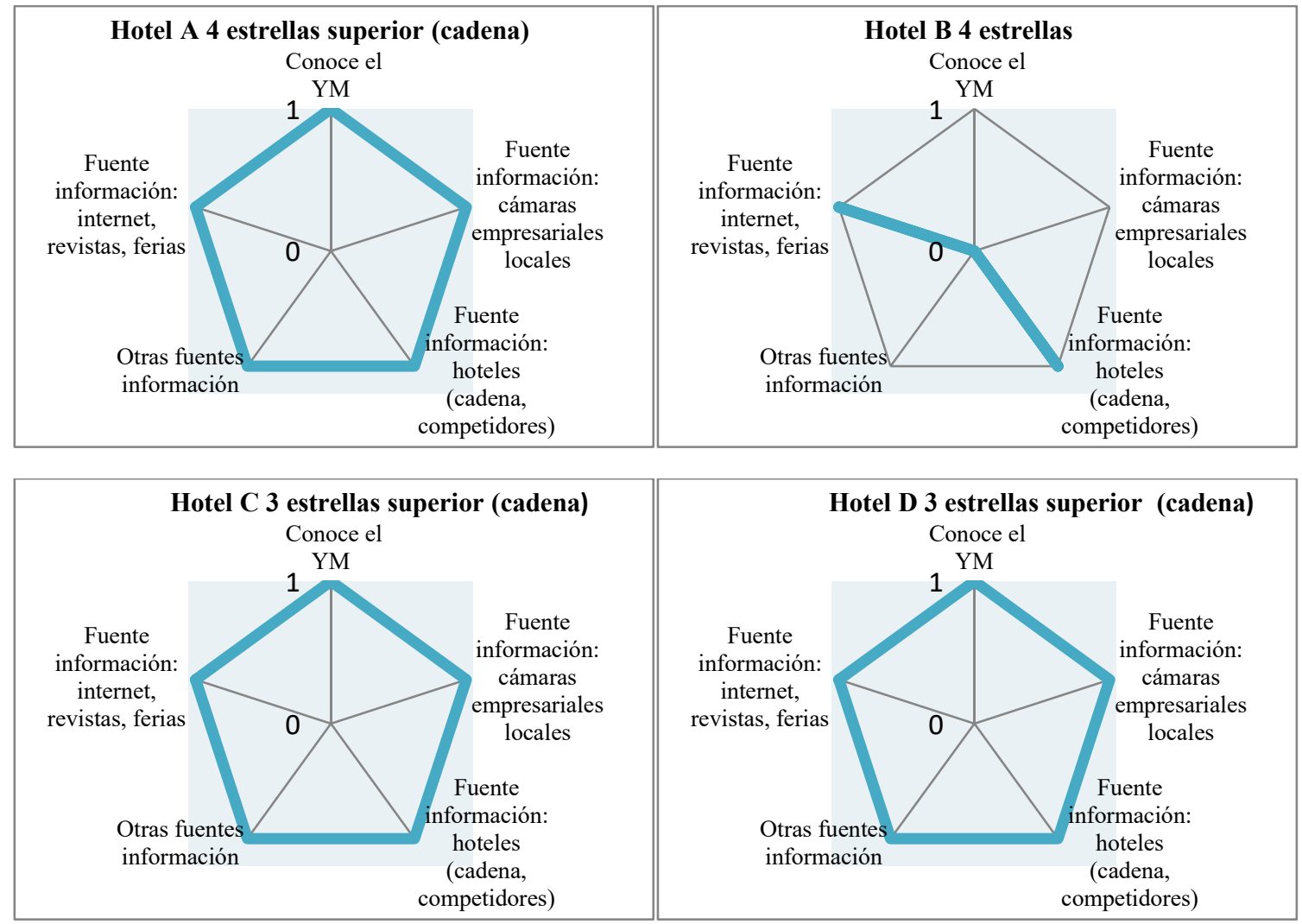

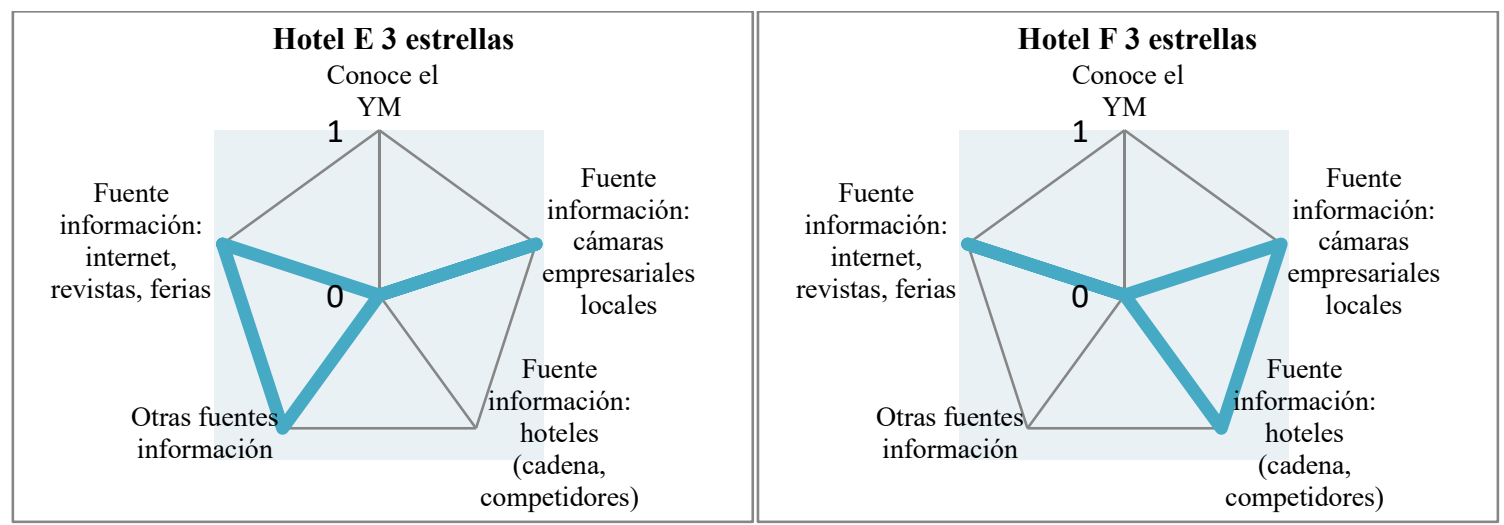




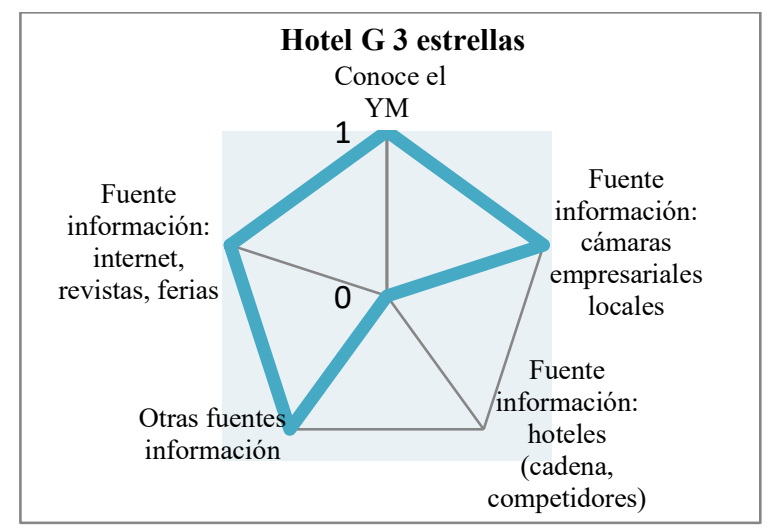

Elaboración propia. Nota: 1 indica que el hotel conoce la técnica YM, usa un determinada fuente de información, 0 en caso contrario.

Respecto a la implementación efectiva de la técnica YM, la Tabla 2 resume algunas características relativas al conjunto de los hoteles entrevistas y la implementación de la técnica YM por parte de los mismos. En este sentido, tres de los hoteles analizados (hoteles A, C y D) declararon usar esta técnica. Estos hoteles son los de mayor categoría dentro de la oferta hotelera de la ciudad y pertenecen a una cadena. El uso de la técnica del YM por parte de estos tres hoteles varía entre diez años y un año a la fecha de la realización de la entrevista. Este conjunto de hoteles segmentan el mercado y consideran a los huéspedes frecuentes y a los grupos para diferenciar tarifas. También aplican la práctica de overbooking.

Tabla 2. Hoteles 3 y 4 estrellas de la ciudad. Implementación técnica YM

\begin{tabular}{|c|c|c|c|c|c|c|c|c|c|c|c|}
\hline HOTEL & $\begin{array}{c}\text { Categorí } \\
\text { a }\end{array}$ & Cadena & $\begin{array}{l}\mathrm{N}^{\circ} \\
\text { Hab- }\end{array}$ & $\begin{array}{l}\text { Segmenta } \\
\text { mercado }\end{array}$ & $\begin{array}{c}\text { Over- } \\
\text { booking }\end{array}$ & $\begin{array}{l}\text { Sistema } \\
\text { reservas }\end{array}$ & $\begin{array}{l}\text { Conoce } \\
\text { YM }\end{array}$ & $\begin{array}{l}\text { Uso } \\
\text { YM }\end{array}$ & $\begin{array}{l}\text { Inicio } \\
\text { uso YM }\end{array}$ & $\begin{array}{l}\text { YM } \\
\text { Soft- } \\
\text { ware }\end{array}$ & $\begin{array}{c}\text { Yield } \\
\text { Mana- } \\
\text { ger }\end{array}$ \\
\hline Hotel A & $\begin{array}{c}4 \\
\text { estrellas } \\
\text { superior }\end{array}$ & Pertenece & 108 & SI & SI & SI & SI & SI & 2008 & SI & $\mathrm{NO}$ \\
\hline Hotel B & $\begin{array}{c}4 \\
\text { estrellas }\end{array}$ & $\begin{array}{c}\text { No } \\
\text { pertenece }\end{array}$ & 100 & SI & SI & SI & $\mathrm{NO}$ & $\mathrm{NO}$ & - & - & - \\
\hline Hotel C & $\begin{array}{c}\text { 3 } \\
\text { estrellas } \\
\text { superior }\end{array}$ & Pertenece & 108 & SI & SI & SI & SI & SI & 2018 & SI & $\mathrm{NO}$ \\
\hline Hotel D & $\begin{array}{c} \\
\text { estrellas } \\
\text { superior }\end{array}$ & Pertenece & 42 & SI & SI & SI & SI & SI & 2018 & SI & $\mathrm{NO}$ \\
\hline Hotel E & $\begin{array}{c}3 \\
\text { estrellas }\end{array}$ & $\begin{array}{c}\text { No } \\
\text { pertenece }\end{array}$ & 80 & SI & NO & SI & $\mathrm{NO}$ & $\mathrm{NO}$ & - & - & \\
\hline Hotel F & $\begin{array}{c}3 \\
\text { estrellas }\end{array}$ & $\begin{array}{c}\text { No } \\
\text { pertenece }\end{array}$ & 44 & SI & SI & SI & $\mathrm{NO}$ & $\mathrm{NO}$ & - & - & \\
\hline Hotel G & $\begin{array}{c}3 \\
\text { estrellas }\end{array}$ & $\begin{array}{c}\text { No } \\
\text { pertenece }\end{array}$ & 16 & SI & NO & SI & SI & NO & - & - & \\
\hline
\end{tabular}

Elaboración propia

A partir de la identificación de los hoteles que implementan efectivamente la técnica YM, se analiza si cuentan con personal idóneo, usan sistemas informáticos adecuados y los inconvenientes percibidos en la aplicación de la técnica. De acuerdo a la Tabla 2, los tres hoteles que aplican la técnica emplean softwares específicos a la actividad hotelera (ARION 
PMS y CLOCL PMS $)^{8}$. Se trata de plataformas digitales de nueva generación para la gestión hotelera y cuentan con una gran cantidad de herramientas, entre ellas la inteligencia en la fijación de tarifas para mejorar la rentabilidad y productividad. Por otra parte, ninguno de los hoteles que implementan la técnica cuenta con la figura del Yield Manager. Por el contrario, el Jefe de ventas y el Jefe de recepción y Gerencia o los propios encargados del hotel destinan parte de su tiempo a la aplicación de esta técnica. Al respecto, es preciso destacar que, dos de los tres hoteles mencionados, los cuales pertenecen a una misma cadena, toman sólo en consideración los parámetros estipulados por el software del YM y los resultados que el sistema determina son los que se utilizan para fijar el precio de las habitaciones. El hotel restante, también se basa en la demanda histórica y actual, los impuestos y estadísticas generales para la fijación de tarifas. Por último, los tres hoteles que emplean la técnica YM no detectaron dificultades en el uso la misma, aunque señalaron que la cultura local en términos de la gran sensibilidad del huésped al cambio en las tarifas constituye una restricción.

Respecto a los hoteles que no usan la técnica YM (hoteles B, E, F y G), sólo uno corresponde a la categoría 4 estrellas y los restantes tres hoteles a la categoría 3 estrellas. De acuerdo a la Tabla 2, a diferencia de aquellos que aplican la técnica YM, la no pertenencia a una cadena es el denominador común entre los hoteles que no emplean esta técnica. Entre estos hoteles, sólo el hotel $\mathrm{G}$ de tres estrellas manifestó conocer la técnica. La fijación de tarifas en este conjunto de hoteles se basa en los costos operativos y la variación del tipo de cambio nominal respecto al dólar. Estos cuatros hoteles, además, segmentan el mercado, en especial entre huéspedes frecuentes. A modo de ejemplo, uno de los hoteles de tres estrellas recibe frecuentemente contingentes de deportistas, motivo por el cual diferencia tarifas por grupos. Por otra parte, el overbooking no es una práctica difundida entre los hoteles que no aplican la técnica.

Si bien la mayoría de los hoteles que no aplican esta técnica manifestaron no tener conocimiento previo sobre la misma, cuando se indicó al entrevistado sobre el concepto y características del YM, estos consideraron que podría ser una opción a implementar y que dicha técnica de fijación de tarifas maximizaría sus ingresos. Sin embargo, también señalaron que la situación económica actual del país (recesión, inflación, incertidumbre), los costos elevados del propio sector y la falta de personal idóneo constituyen restricciones para su pronta implementación.

Por último, de acuerdo a la Tabla 2, es preciso destacar que, todos los hoteles entrevistados, segmentan el mercado y cuentan con un sistema de reservas, aplicando la mayoría prácticas de overbooking. De este modo, el conocimiento sobre YM y la aplicación efectiva o potencial de esta técnica parece no tener relación con las prácticas mencionadas.

\section{Discusión de los resultados y reflexiones finales}

La innovación, como respuesta a un escenario de creciente competencia y dinamismo, en el sector hotelero se encuentra ampliamente ligada al uso de las TICs. De este modo, distintos autores califican al sector como intensivo en información Entre las innovaciones asociadas al uso de las TICs, se encuentra la técnica Yield Management (YM), la cual resulta adecuada para los sectores que operan con capacidad relativamente fija, inventario perecedero, posibilidad de vender el producto anticipadamente. Entre las distintas dimensiones de análisis de la técnica YM, el presente trabajó se centró específicamente en el uso de esta técnica para la fijación de

\footnotetext{
${ }^{8}$ La sigla PMS significa sistema de gestión de la propiedad (Property Management System). La sigla CRS refiere a sistema central de reservas (Central Reservation System).
} 
tarifas. El estudio procuró conocer el grado de difusión de la técnica YM entre los hoteles de 3 y 4 estrellas de la ciudad de Bahía Blanca, Argentina. Esta localidad urbana de tamaño poblacional medio constituye un centro comercial, financiero y de servicios de influencia regional, nodo de transporte y comunicaciones. La ciudad cuenta, además, con una variada oferta de alojamientos. Los turistas se hospedan en los hoteles de 3 y 4 estrellas analizados, principalmente por razones laborales, cuestiones vinculadas a la demanda de servicios de salud y la realización de trámites en los organismos político-administrativos de la ciudad.

Tal como se indicó en la Introducción, se espera que el presente estudio, de carácter exploratorio y descriptivo, sirva para incrementar el conocimiento empírico sobre el grado de difusión de la técnica YM y los obstáculos asociados a su implementación, específicamente, en hoteles que no pertenecen a grandes cadenas internacionales y se encuentran localizados en países de menor grado de desarrollo. A modo de síntesis, los resultados encontrados 1) dan cuenta de la importancia de la pertenencia a una estructura organizativa de mayor complejidad, aun cuando se trate de cadenas de origen y alcance nacional; 2) refuerzan sobre la falta de recursos humanos especializados como característica sectorial y; 3 ) ofrecen evidencia sobre la importancia factores relativos al contexto macroeconómico especifico al país bajo estudio. Este último resultado marca una diferencia en relación a estudios previos. A continuación se detallan tales resultados.

De acuerdo a la información primaria relevada a través de entrevistas, se obtuvieron resultados en línea con estudios previos sobre YM en hotelería (Chávez Miranda et al., 2014; López-Fernández et al., 2009; Chávez Miranda y Ruiz Jiménez, 2003; Kimes, 2017). En este sentido, en primer lugar, factores organizacionales, en términos de pertenencia a una cadena, y de demanda, en términos de categoría, resultan relevantes. Los hoteles que declararon conocer la técnica YM e implementar la misma son aquellos de mayor categoría (4 y 3 estrellas superior) y pertenecientes a una cadena hotelera. Los hoteles de categoría 3 estrellas y que, coincidentemente no pertenecen a una cadena, no aplican la técnica YM y no cuentan con conocimiento previo sobre la misma. En estos últimos hoteles, la fijación de tarifas se basa en variables monetarias (variación de los costos en función de la inflación y el tipo de cambio nominal).

Un segundo resultado se asocia a la profesionalización de los recursos humanos. La necesidad de contar con recursos humanos calificados surge en el estudio propuesto, por un lado, al analizar el acceso a información y conocimiento externo. En línea con el estudio de Chávez Miranda y Ruiz Jiménez (2003), todos los entrevistados declararon que usan fuentes disponibles (revistas, informes, Internet) para actualizarse sobre nuevas técnicas. De todos modos, el uso de fuentes de información en combinación con el desconocimiento sobre la técnica YM por parte de los hoteles sugiere indagar en la capacidad de absorción (Cohen y Levinthal, 1990) de los mismos. De acuerdo Cohen y Levinthal (1990) la capacidad de absorción entendida como la capacidad para identificar, asimilar y explorar información externa a la firma se deriva, en parte, de la capacitación de los recursos humanos. Al respecto, es preciso destacar que se trata de un sector con un bajo nivel de profesionalización del personal. Entre las restricciones a la innovación en hoteles, Fernández et al. (2011) señalan que el turismo va muy por detrás de otros sectores con respecto a la calificación de sus recursos humanos debido a una rotación excesiva, mayor inestabilidad, movilidad, contratos de trabajo de baja calidad. Este limitante adquiere especial relevancia si se tiene en cuenta que las innovaciones de tipo incremental provienen del personal que se encuentra en contacto directo con el huésped durante el proceso de producción y consumo del servicio de alojamiento (Moliner, Azorín, Guilló, Ortega y Gamero, 2019). Por otro lado, en relación específica a innovaciones de tipo back- 
office tal como la adopción de la técnica YM, el surgimiento de numerosos canales de distribución, vuelve más complejo el proceso de toma de decisiones sobre fijación de tarifas y requiere de personal calificado y afectado específicamente a tal tarea. Sin embargo, la ausencia de la figura del Yield Manager es un resultado que se deriva del estudio de caso propuesto. Los hoteles de 3 y 4 estrellas superior adquieren tecnología incorporada en softwares específicos al sector, sin embargo, no cuentan con personal dedicado exclusivamente a la operación de la técnica. Tal como se indicó este resultado es propio de los hoteles de menor tamaño (Ferguson y Smith, 2014).

Un tercer resultado derivado del estudio de caso propuesto refiere a las limitantes que afrontan los hoteles respecto a la aplicación potencial y el uso efectivo de la técnica YM. En este punto, se indagó sobre los motivos por los cuáles los hoteles no emplean la técnica. Si bien, el desconocimiento es el factor principal, los entrevistados reconocen que variables relativas al entorno macro (incertidumbre, inflación, recesión) afectan la potencial aplicación de nuevas técnicas. Este tipo de variables también influyen en las decisiones de inversión en innovación en el sector industrial de Argentina (MINCYT, 2015). En sentido contrario, en estudios sobre innovación en hotelería (López-Fernández et al., 2009), y específicamente, sobre el uso de la técnica YM (Chávez Miranda et al., 2014; Chávez Miranda y Ruiz Jiménez, 2003), los factores de contexto macro tienen una influencia moderada respecto a factores internos a la empresa. Por otra parte, los hoteles que emplean la técnica YM indican sobre la percepción negativa de los huéspedes ante cambios en las tarifas, aspecto ampliamente abordado en la literatura (Kimes, 2002; Choi y Mattila, 2004, entre otros). A este último factor, se suma, tal como se indicó, la falta de personal asignado específicamente a la tarea de operar los sistemas de gestión y analizar sus resultados.

Por último, a modo de lineamiento futuro, dadas las características de la ciudad de Bahía Blanca, donde el turismo por ocio no es el principal motivo de su ocupación hotelera, sería de interés la realización de un estudio similar en ciudades turísticas con el objetivo de confirmar los hallazgos de este trabajo. Sin embargo, dada la inexistencia de otras investigaciones en el área tanto a nivel nacional como en relación a otros países de América Latina, este trabajo puede ser un primer disparador para profundizar el conocimiento del sector y ayudar a la toma de decisiones en un aspecto tan fundamental para la empresa hotelera como es la fijación de tarifas. Asimismo, los resultados encontrados sugieren indagar con mayor profundidad en los factores condicionantes de la innovación en hoteles, extendiendo el análisis hacia distintos tipos de innovación más allá de la innovación de tipo back-office tratada en el presente trabajo.

\section{Referencias}

Anderson, C. K., \& Xie, X. (2010). Improving hospitality industry sales: Twenty-five years of revenue management. Cornell Hospitality Quarterly, 51(1), pp. 53-67. DOI: $10.1177 / 1938965509354697$

Benckendorff, P., Sheldon, P. \& Fesenmaier, D.R. (2019). Tourism information technology, $3^{\text {rd }}$ Edition, Oxford, UK: CABI

Cohen, W.M., \& Levinthal, D.A. (1990). Absorptive capacity: A new perspective on learning and innovation. Administrative Science Quarterly, pp. 128-152. DOI: 10.2307/2393553. 
Comisión Europea (1997). Yield management in small and medium sized enterprises in the tourism industry. European Commission - General Report. European Commission. DirectorateGeneral XXIII, Tourism Unit, Brussels, Luxembourg.

Chávez Miranda, M.E., Ruiz Jiménez, A., \& Pérez Díez de los Ríos, J.L. (2014). Factores críticos que dificultan la aplicación de Revenue Management en hoteles urbanos. International Journal of World of Tourism, 1(1), pp. 26-41.

Chávez Miranda, M.E., \& Ruiz Jiménez, A. (2005). Yield Managerment en Hotelería. Estudio de su aplicación en Sevilla. Ayuntamiento de Sevilla.

Cheraghi, S.H., Dadashzadeh, M., \& Venkitachalam, P. (2010). Revenue management in manufacturing: a research landscape. Journal of Business \& Economics Research (JBER), 8(2). https://doi.org/10.19030/jber.v8i2.673

Choi, S., \& Kimes, S.E. (2002). Electronic distribution channels' effect on hotel revenue management. Cornell Hotel and Restaurant Administration Quarterly, 43(3), pp. 23-31. https://doi.org/10.1177/0010880402433002

Choi, S., \& Mattila, A.S. (2004). Hotel revenue management and its impact on customers' perceptions of fairness. Journal of Revenue and Pricing Management, 2(4), pp. 303-314. https://doi.org/10.1057/palgrave.rpm.5170079

Diez, J.I. (2008). Organizaciones, redes, innovación y competitividad territorial: análisis del caso Bahía Blanca. Redes. Revista Hispana para el Análisis de Redes Sociales, (14), pp. 1 -31.

Emeksiz, M., Gursoy, D., \& Icoz, O. (2006). A yield management model for five-star hotels: Computerized and non-computerized implementation. International Journal of Hospitality Management, 25(4), pp. 536-551. https://doi.org/10.1016/j.ijhm.2005.03.003

Erdem, M., \& Jiang, L. (2016). An overview of hotel revenue management research and emerging key patterns in the third millennium. Journal of Hospitality and Tourism Technology, 7(3), pp. 300-312. https://doi.org/10.1108/JHTT-10-2014-0058

Fernández, J.I.P., Cala, A.S., \& Domecq, C.F. (2011). Critical external factors behind hotels' investments in innovation and technology in emerging urban destinations. Tourism Economics, 17(2), pp. 339-357. https://doi.org/10.5367/te.2011.0033

Ferguson, M., \& Smith, S. (2014). The changing landscape of hotel revenue management and the role of the hotel revenue manager. Journal of Revenue and Pricing Management, 13(3), pp. 224-232. https://doi.org/10.1057/rpm.2014.11

González Serrano, L., Talón, P. y Figueroa, C. (2004), "La optimización de la gestión en los servicios turísticos a través del Yield Management", TuriTec. V Congreso Turismo y Tecnologías de la Información y las comunicaciones, pp.129-147, [disponible en: https://www.researchgate.net/publication/267259640_La_Optimizacion_de_la_Gestion_en_lo s_Servicios_Turisticos_a_Traves_del_Yield_Management].

Gorenstein, S., Geymonat, A., Landriscini, G., Hernández, J., Preiss, O., Domeett, G., y Pasciaroni, C. (2010). Una revisión y algunas preguntas sobre ciudades intermedias. 
Explorando funciones en ciudades pampeanas y norpatagónicas. XI Seminario Internacional RII de Investigadores sobre Territorio y Globalización. Universidad Nacional de Cuyo. Mendoza, pp. 28-30.

Heo, C. Y., \& Lee, S. (2011). Influences of consumer characteristics on fairness perceptions of revenue management pricing in the hotel industry. International Journal of Hospitality Management, 30(2), pp. 243-251. https://doi.org/10.1016/j.ijhm.2010.07.002

INDEC - Instituto Nacional de Estadística y Censos de Argentina (2019). Encuesta de Ocupación Hotelera. Cifras estimadas a abril de 2019. Disponible en: https://www.indec.gob.ar/uploads/informesdeprensa/eoh_06_1950E8C2111D.pdf

Ivanov, S. (2014). Hotel Revenue Management: From Theory to Practice. Varna: Zangador Ltd.

Ivanov, S.H. (2006). Management of overbookings in the hotel industry-basic concepts and practical challenges. Tourism Today, 6, pp. 19-32.

Johnson, E. (2006). Yielding results: removing the human factor from pricing may boost revenue. Journal of Property Management, 71(1), pp. 42-48.

Jones, P. (2000). Defining yield management and measuring its impact on hotel performance. En: Ingold, A. et al. (eds), Yield Management Strategies for the Service Industries (pp.85-97), London: Continuum.

Kimes, S.E. (2017). The future of hotel revenue management. Cornell Hospitality Report, 17(1), pp. 3-10.

Kimes, S.E. (2002). Perceived fairness of yield management. Cornell Hotel and Restaurant Administration Quarterly, 43(1), pp. 21-30. https://doi.org/10.1177/0010880402431002

Kimes, S.E. (1989). The basics of yield management. Cornell Hotel and Restaurant Administration Quarterly, 30(3), pp. 14-19. https://doi.org/10.1177/001088048903000309

Kimes, S.E., \& Chase, R.B. (1998). The strategic levers of yield management. Journal of Service Research, 1(2), pp. 156-166. https://doi.org/10.1177/109467059800100205

Kimes, S. E., \& Wirtz, J. (2003). Has revenue management become acceptable? Findings from an international study on the perceived fairness of rate fences. Journal of Service Research, 6(2), pp. 125-135. https://doi.org/10.1177/1094670503257038

Law, R., \& Jogaratnam, G. (2005). A study of hotel information technology applications. International Journal of Contemporary Hospitality Management, 17(2), pp. 170180. https://doi.org/10.1108/09596110510582369

Lehr, I. L. (2018). Alojamientos turísticos en la ciudad de Bahía Blanca. Tesina de Licenciatura en Turismo, Bahía Blanca: Universidad Nacional del Sur.

Lieberman, W.H. (1993). Debunking the myths of yield management. Cornell Hotel and Restaurant Administration Quarterly, 34(1), pp. 34-41. https://doi.org/10.1177/001088049303400107 
Lopes, T.S., \& de Viera, E.V. Compreensões sobre a prática do Yield Management na hotelaria: um estudo de caso comparativo em três hotéis de categoria express em Porto Alegre-RS. FólioRevista Científica Digital-Jornalismo, Publicidade e Turismo, 5(1), pp. 54-72.

López-Fernández, M.C., Serrano-Bedia, A. M., \& Gómez-López, R. (2009). La decisión de innovar de las empresas turísticas: un análisis empírico de la industria hostelera. Investigaciones Europeas de Dirección y Economía de La Empresa, 15(3), pp. 169-182. https://doi.org/10.1016/S1135-2523(12)60107-5

Luciani, S. (1999). Implementing yield management in small and medium sized hotels: An investigation of obstacles and success factors in Florence hotels. International Journal of Hospitality Management, 18(2), pp. 129-142.

Lundvall, B.Å. (Ed.). (2010). National Systems of Innovation: Toward a Theory of Innovation and Interactive Learning (Vol. 2). London-New York: Anthem Press.

MINCYT- Ministerio de Ciencia, Tecnología e Innovación de Argentina (2015). Encuesta Nacional de Dinámica del Empleo y la Innovación. Resultados Período de cobertura: 20102012.

Nieves, J., \& Segarra-Ciprés, M. (2015). Management innovation in the hotel industry. Tourism Management, 46, pp. 51-58. https://doi.org/10.1016/j.tourman.2014.06.002

OECD (Organization for Economic Cooperation and Development)/Eurostat 2005. Guidelines for Collecting and Interpreting Innovation Data - The Oslo Manual, 3rd edn. Paris: OECD.

O'Connor, P., \& Murphy, J. (2008). Hotel yield management practices across multiple electronic distribution channels. Information Technology \& Tourism, 10(2), pp. 161-172. https://doi.org/10.3727/109830508784913103

Okumus, F. (2004). Implementation of yield management practices in service organisations: empirical findings from a major hotel group. The Service Industries Journal, 24(6), pp. 65-89. https://doi.org/10.1080/0264206042000299185

Orfila-Sintes, F., \& Mattsson, J. (2009). Innovation behavior in the hotel industry. Omega, 37(2), pp. 380-394. https://doi.org/10.1016/i.omega.2007.04.002

Orkin, E.B. (1988). Boosting your bottom line with yield management. Cornell Hotel and Restaurant Administration Quarterly, 28(4), pp. 52-56. https://doi.org/10.1177/001088048802800416

Moliner, J. P., Azorín, J. F. M., Guilló, J. J. T., Ortega, E. M. P., \& Gamero, M. D. L. (2019). Sostenibilidad, calidad, innovación y ventaja competitiva en el sector hotelero: Un estudio de caso. Revista Internacional de Turismo, Empresa y Territorio, 3(1), pp. 1-21.

https://doi.org/10.21071/riturem.v3i1.11474

Sigala, M. (2003). The information and communication technologies productivity impact on the UK hotel sector. International Journal of Operations \& Production Management, 23(10), pp. 1224-1245. https://doi.org/10.1108/01443570310496643 
Smith, B. C., Leimkuhler, J. F., \& Darrow, R. M. (1992). Yield management at American airlines. Interfaces, 22(1), pp. 8-31. https://doi.org/10.1287/inte.22.1.8

Vander Tuin, V. (2019). La fijación de tarifas en los hoteles de tres y cuatro estrellas de la ciudad de Bahía Blanca: análisis del uso de la técnica de Yield Management. Tesina de Licenciatura en Turismo, Bahía Blanca: Universidad Nacional del Sur.

Wirtz, J., Kimes, S. E., Theng, J. H. P., \& Patterson, P. (2003). Revenue management: resolving potential customer conflicts. Journal of Revenue and Pricing Management, 2(3), pp. 216-226. https://doi.org/10.1057/palgrave.rpm.5170068

Yin, R. K. (1981). The case study crisis: Some answers. Administrative Science Quarterly, 26(1), pp. 58-65. DOI: 10.2307/2392599 